\title{
Possibility of waveguide formation on organic nonlinear crystal methyl para-hydroxy benzoate using high energy ion irradiation
}

\author{
P. Sreeramana Aithal ${ }^{a}$, H.S. Nagaraja ${ }^{b}$, P. Mohan Rao ${ }^{\text {b.* }}$, V.P.N. Nampoori ${ }^{c}$, \\ C.P.G. Vallabhan ${ }^{\mathrm{c}}$, D.K. Avasthi ${ }^{\mathrm{d}}$ \\ ${ }^{a}$ Department of Electronics, NSAM College, Nitte-574 110, India \\ ${ }^{\mathrm{b}}$ Department of Physics, Mangalore University, Mangalagangotri-574 199, India \\ ' Laser division, Department of Physics, Cochin University of Science and Technology, Cochin-682 022, India \\ 'Nuclear Science Centre, Arun Asaf Ali Marg, PB 10502, New Delhi-110 067, India
}

Received 5 July 1996; revised form received 10 February 1997

\begin{abstract}
Organic nonlinear optical single crystals of Methyl para-Hydroxy Benzoate (MHB) have been grown using gel-solution technique. These crystals are cut along $z$-axis and are bombarded with $\mathrm{Ag}^{14+}$ ions of energy $100 \mathrm{MeV}$. The results show an increase in refractive index at the ion irradiated region. The dielectric constant of the irradiated crystal is increased more than 15 times compared to that of a nonirradiated crystal. The result of these changes and comparative study of second harmonic generation (SHG) efficiency before and after irradiation is discussed.
\end{abstract}

\section{Introduction}

A basic element of future nonlinear optical or optoelectronic devices and integrated structures will most probably consist of an optical waveguide configuration as first proposed by Miller [1]. Waveguide configurations offer an effective approach to accomplish the operations like modification, switching, amplification, frequency conversion. light beam splitting and combination, etc. [2].

High power densities can be produced in waveguides with less than milliwatt optical powers generated by laser diodes. Diffractionless propagation over relatively long distances required for optical and electrical control of devices based on the light or voltage dependent refractive index change is easily obtained in optical waveguides [3]. The refractive index changes themselves are thus enhanced owing to the field confinement over a guiding distance. Therefore, for example, the use of relatively weak optical nonlinearities become possible.

An optical waveguide comprises a layer or stripe which has high refractive index relative to its surroundings [4]. Any fabrication technique must therefore have the effect of either raising the index of the guide or lowering that of surroundings. There are several conventional methods of producing optical waveguides, but they do not have any

\footnotetext{
"Corresponding author. Fax: +91-824-742367; email: root@mnglr.emet.in
}

general applicability and there are certain disadvantages in each case.

Topographically produced ridge waveguides are perhaps close to a theoretical ideal. Unfortunately, the physical problems of producing ridges are not insignificant and low scattered structures are not easily obtained. The epitaxial growth of a high index layer on a low index substrate is often successful for planar waveguide production, but for strip guides masking must be used either during deposition or during subsequent etching.

The more recently introduced technique of ion implantation has several advantages of being a generally applicable process [5]; it can be performed at ambient or even at lower temperatures, it has no side diffusion problem; and it can, to some extent, provide a degree of index profile tailoring to suit various applications.

\section{Ion implanted waveguides}

The effects of ion implantation which may result in refractive index change are twofold. Firstly, the energy deposited by the ion induces physical changes in the substrate. These may only be ionization effects, or, they may also be structural changes occurring either as isolated point defects or as large regions of phase reconstruction. These structural changes occur to some extent along the entire range of the ion, but the major effect dominates in the low velocity region near to the end of the track. The 
second effect of ion implantation with possible refractive index consequences is a chemical one, due to the actual presence of the implanted ion and this therefore exists only over the region where the ions come to rest [6]. The result of the ion passage is either ionization (in electronic stopping region) or structural defects (in nuclear stopping region). These affect the refractive index due to the changes in physical density, bond polarizability and various other structural effects. The ionization may have a minor bearing on the bond polarizability factor, but any major index change will be due to physical movement of the lattice atoms. This is expected to produce an expansion in ordered structures (crystals) and will give a negative $\Delta n$, but it may be a compaction (e.g., in some glasses [7]) giving positive $\Delta n$. In general, in the surface region, the change may be positive or negative due to the combination of damage, ionization and diffusion effects. In some materials (e.g., fused silica, BGO) only an increase in index is apparent [8,9].

Ion implanted waveguides have now been used to produce SHG in crystals of $\mathrm{SiO}_{2}, \mathrm{KTP}, \mathrm{KNbO}_{3}$ and $\mathrm{LBO}$ [10-12]. Conversion efficiencies of nearly $30 \%$ have been quoted for $\mathrm{KTP}$ and $\mathrm{KNbO}_{3}$ and at the same time, the nonlinearity of these materials has not been appreciably reduced by the ion implantation.

It is interesting to study the similar effects due to high energy ion irradiation in which instead of implantation, the irradiated ion scatters out due to its tremendous amount of energy. These irradiated ions may produce structural modifications, in the crystal, producing the changes in refractive index and other properties.

In this paper, the effect of high energy ion irradiation $\left(\mathrm{Ag}^{14+}\right)$ on refractive index, dielectric constant and other properties of an efficient organic nonlinear crystal Methyl $\mathrm{p}$-Hydroxy Benzoate (MHB) is reported. This is the first attempt to examine the possibility of waveguide formation due to heavy ion irradiation in case of organic nonlinear optical crystals.

\section{Experimental}

\subsection{Crystal growth and characterization}

The commercially available methyl p-hydroxy benzoate (MHB) is purified by means of repeated recrystallization. To grow single crystals of $\mathrm{MHB}$, a new variation in gel technique named as Gel-solution technique [13] is employed. In this technique sodium meta-silicate of specific gravity 1.04 is mixed with glacial acetic acid to prepare the gel of required $\mathrm{pH}$. After setting the gel, the solution of MHB in acetone is added slowly without disturbing the gel structure. The gel $\mathrm{pH}$ is maintained at 4.2 . The growth process involved the controlled diffusion and evaporation of the solvent producing necessary supersaturation for the growth of crystals on the surface of the gel. The growth experiment was carried out in test tubes of length $20 \mathrm{~cm}$ and of diameter $25 \mathrm{~mm}$ at ambient temperature. The pure transparent crystals of size $22 \times 16 \times 9 \mathrm{~mm}^{3}$ were obtained. In order to further increase the size of the crystals, these crystals were used as seed crystals and larger crystals were grown by solvent evaporation solution technique.

The purity of grown crystals is verified by $\mathrm{X}$-ray powder diffraction, mass spectroscopy and NMR techniques. From single crystal $X$-ray diffraction studies, it is found that $\mathrm{MHB}$ crystallizes in monoclinic, $C_{c}$ space group with unit cell dimensions $a=13.568 \AA, b=16.959$ $\AA, c=12.458 \AA$ [14]. The optical absorption spectrum of MHB shows the cut-off wavelength as $210 \mathrm{~nm}$. The mechanical properties are studied by means of microhardness measurements and the observed value of vicker's hardness number is $43.12 \mathrm{~kg} / \mathrm{mm}^{2}$ for $50 \mathrm{~g}$ of applied indentation load along $z$-axis.

\subsection{Ion irradiation}

The MHB crystals are cut into plates of $2 \mathrm{~mm}$ thickness, perpendicular to $z$-axis and polished. $\mathrm{Ag}^{14+}$ ions of $100 \mathrm{MeV}$ from the $15 \mathrm{MV}$ Pelletron [15] at Nuclear Science Centrc (NSC), Delhi, were incident on the sample mounted inside a $1.5 \mathrm{~m}$ diameter scattering chamber [16]. The beam current was about 4 particle nano Ampere ( $\mathrm{pn} A=$ current in nanoAmpere/charge state of the ion). The sample was kept at an angle of $30^{\circ}$ with respect to the beam direction, so that hydrogen $(\mathrm{H})$ ion measurement during ion irradiation can be made. The vacuum in the chamber during the experiment was $8 \times 10^{-7} \mathrm{mbar}$.

\section{Results and discussion}

\subsection{Determination of hydrogen $(H)$ concentration}

$\mathrm{H}$ depth-profiling was carried out by Elastic Recoil Detection Analysis (ERDA) [17,18]. A surface barrier detector having a depletion depth of $1 \mathrm{~mm}$ was used to detect $\mathrm{H}$ recoils at an angle of $46^{\circ}$ with respect to the beam direction. The detector had a $26 \mu \mathrm{m}$ polypropylene foil to stop unwanted heavier recoils. The details of the experiment and analysis are given elsewhere $[19,20]$. The incident $100 \mathrm{MeV} \mathrm{Ag}$ ions have a range of $16 \mu \mathrm{m}$ in the crystal as determined by Transport of Ion in Matter (TRIM) calculation. The ions, however, get embedded at the depth of $8 \mu \mathrm{m}$ due to $30^{\circ}$ tilt of the sample. It is observed that $\mathrm{H}$ concentration decreased during ion irradiation. The $\mathrm{H}$ loss was monitored as a function of dose as shown in Fig. 1. 


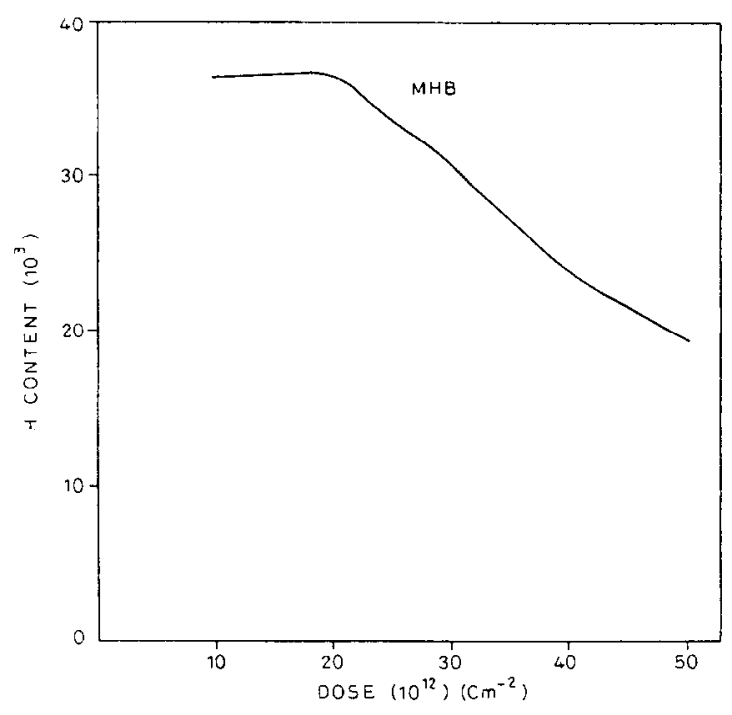

Fig. 1. Plot of yield versus dose of $\mathrm{Ag}^{14+}$ ions.

At an ion irradiation dose above $10 \times 10^{12} \mathrm{~cm}^{-2}$, due to enormous loss of $\mathrm{H}$, the sample was darkened at the beam spot. The $\mathrm{H}$ measurement indicates that at higher dose rate, the residual material is $\mathrm{H}$ deficient or carbon rich. All further characterizations are carried out at low dose $\left(10 \times 10^{12} \mathrm{~cm}^{-2}\right)$ irradiated crystals.

\subsection{Studies on dielectric properties}

The capacitance of the crystals are measured at different frequencies using a HP Impedance/gain-phase analyzer (Model4194 A) at room temperature. The dielectric constants of these crystals are compared with those of non irradiated crystals as given in Table 1 .

The dielectric constant is found to increase by nearly 15 times from the values obtained for the nonirradiated crystals. Further, no considerable variation is observed in dielectric constant values with increase in applied frequency before and after ion irradiation. The drastic increase in dielectric constant is probably due to the defects generated around the range of the embedded ions and the modification induced in the region above it, up to the

Table 1

Comparison of dielectric constants of MHB.

\begin{tabular}{rll}
\hline Frequency $(\mathrm{Hz})$ & \multicolumn{2}{l}{ Dielectric constant } \\
\cline { 2 - 3 } & $\begin{array}{l}\text { before } \\
\text { irradiation }\end{array}$ & $\begin{array}{l}\text { after } \\
\text { irradiation }\end{array}$ \\
\hline $10 \mathrm{~K}$ & 6.16 & 94.88 \\
$50 \mathrm{~K}$ & 6.30 & 94.30 \\
$100 \mathrm{~K}$ & 6.26 & 93.33 \\
$500 \mathrm{~K}$ & 6.23 & 92.48 \\
$1 \mathrm{M}$ & 6.33 & 91.41 \\
\hline
\end{tabular}

Table 2

Comparison of refractive index values.

\begin{tabular}{lll}
\hline Crystal & $n$ before irradiation & $n$ after irradiation \\
\hline MHB & 1.738 & 1.784 \\
\hline
\end{tabular}

surface due to the electronic excitation which also causes $\mathrm{H}$ loss.

It is known that the electro-optic coefficient $\left(r_{\text {eff }}\right)$ is directly proportional to the dielectric constant of the material. Hence it may be argued that the electro-optic coefficient of MHB enhances due to high energy ion irradiation.

\subsection{Refractive index measurements}

The linear refractive index on the surface of the crystal at the region of irradiation is measured using Brewster's angle method. He-Ne laser light of wavelength 0.6328 $\mu \mathrm{m}$ is used as incident light and the reflected light is scanned by means of a power meter. The results are tabulated in Table 2.

The result shows an increase in refractive index of MHB due to ion irradiation. In case of some polymers like PMMA and polystyrene, the increase in refractive index is observed due to implantation of low energy ions [21-23]. This increase in refractive index after heavy ion irradiation in organic MHB crystal proves the ability of heavy ion irradiation to fabricate planar waveguides on this type of crystals. However, the studies on the performance of the waveguide as such have to be studied further.

\subsection{Second harmonic generation conversion efficiency measurement}

To study the effect of ion irradiation and subsequent disordering of crystal lattice on nonlinear properties of $\mathrm{MHB}$, the second harmonic generation efficiency of irradiated crystal is compared with that of nonirradiated crystal. The ion irradiated crystals are placed on a goniometer and the Q-switched Nd-YAG laser beam of wavelength 1.064 $\mu \mathrm{m}$ (10 ns pulse width, $10 \mathrm{~Hz}$ repetition rate) is directed on irradiated surface (phase matching direction). The SHG

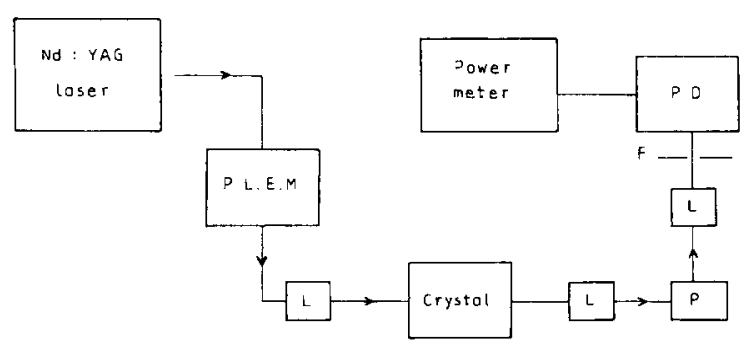

Fig. 2. Schematic diagram of SHG efficiency measurement system. 
Table 3

Comparison of SHG conversion efficiency.

\begin{tabular}{lll}
\hline Input energy $(\mathrm{mJ})$ & \multicolumn{2}{l}{ Output power $(\mathrm{W})$} \\
\cline { 2 - 3 } & $\begin{array}{l}\text { before } \\
\text { irradiation }\end{array}$ & $\begin{array}{l}\text { after } \\
\text { irradiation }\end{array}$ \\
\hline 20 & 0.0084 & 0.0080 \\
40 & 0.0196 & 0.0184 \\
60 & 0.0496 & 0.0457 \\
80 & 0.0968 & 0.0940 \\
100 & 0.1350 & 0.1252 \\
120 & 0.1970 & 0.1892 \\
140 & 0.2705 & 0.2527 \\
160 & 0.3300 & 0.3010 \\
180 & 0.3877 & 0.2500 \\
200 & 0.3804 & 0.2175 \\
\hline
\end{tabular}

radiation of wavelength $0.532 \mu \mathrm{m}$ emerging out from opposite surface is monitored using a thermoelectric detector and power meter assembly. The schematic diagram of the measurement system is given in Fig. 2. The input energy is measured by means of an online energy meter. The fundamental wavelength at output is filtered by means of a prism and an IR filter. By increasing the input energy in $\mathrm{mJ}$, the output SHG power is determined. The comparison of SHG conversion efficiencies of irradiated and nonirradiated crystals is given in Table 3.

The results show that nonlinearity and conversion efficiency of MHB is not affected considerably due to heavy ion irradiation at low input energy of the laser beam. However, with increase in input energies beyond $160 \mathrm{~mJ}$, the irradiated crystal gets damaged earlier compared to nonirradiated crystal. This indicates that high energy ion irradiation lowers the laser damage threshold of these crystals.

\section{Conclusions}

The high energy heavy ion irradiation of organic nonlinear crystal MHB, increased the dielectric constant as well as refractive index at the region of irradiation. Furthermore the structural defect produced has not affected the nonlinear property i.e., second harmonic conversion efficiency of the crystal for low input power of laser light. The increase in refractive index at the region of ion irradiation can be successfully utilized for the waveguide fabrication in MHB crystals.

\section{Acknowledgement}

The present work is partially supported by a project from Department of Science and Technology, Government of India, which is gratefully acknowledged.

\section{References}

[1] S.E. Miller, Bell Syst. Tech. J. 48 (1969) 2059.

[2] M. Cada, J. He, R. Normandin and S. Janz, Int. J. Nonlinear Opt. Phys. 3 (1994) 169.

[3] A. Gover and A. Yariv, J. Appl. Phys. 45 (1974) 2596.

[4] P.K. Tien, Rev. Mod. Phys. 49 (1977) 361.

[5] P.D. Townsend, P.J. Chandler and L. Zhang, Optical Effect of ion Implantation (Cambridge University Press, Cambridge, 1994)

[6] F.P. Strohkendl, P. Gunter and R. Irmscher, J. Appl. Phys. 69 (1991) 84 .

[7] A.B. Faik, P.J. Chandler and R. Webb, Radiat. Eff. 98 (1986) 233.

[8] S.M. Mahdavi, G. Lifante and P.D. Townsend, Nucl. Instr. and Meth. B 65 (1992) 251.

[9] P.J. Chandler and P.D. Townsend, Nucl. Instr. and Meth. B 80/81 (1993) 1135.

[10] L. Babsail and P.D. Townsend, Appl. Phys. Lett. 59 (1991) 384.

[11] D. Fluck and P. Gunter, Opt. Commun. 90 (1992) 304.

[12] D. Fluck, D.J. Moll, P. Gunter and C. Buchal, Electron. Lett. 28 (1992) 1092.

[13] P. Srecramana Aithal and P. Mohan Rao, J. Cryst. Growth 153 (1995) 60

[14] Lin Xianti, Jiegou Huaxue 2 (1983) 91.

[15] G.K. Mehta and A.P. Patro, Nucl. Instr. and Meth. A 268 (1988) 334.

[16] D.K. Avasthi, A. Tripathi, D. Kabiraj, S. Venkataramanan and S.K. Dutta, Internal report NSC/TR/DKA/92/16.

[17] J. L'Ecuyer, C. Brassard, C. Cardinal and B. Terreault, Nucl. Instr. and Meth. 149 (1978) 271.

[18] J. L’Ecuyer, C. Brassard, C. Cardinal, J. Chabbal, L. Deschenes, J.P. Lalrie, B. Terranlt, J.G. Martil and R. St.-Jacques, J. Appl. Phys. 47 (1976) 381.

[19] D.K. Avasthi, M.G. Acharya, R.D. Tarey, L.K. Malhstra and G.K. Mehta, Vacuum 46 (1995) 265.

[20] D.K. Avasthi, D. Kabiraj, Jaipal, G.K. Mehta, H.C. Barshilia, Somna Sha, B.R. Mehta and V.D. Vankar, Vacuum 46 (1995) 633.

[21] W.L. Brown, Nucl. Instr. and Meth. B 37/38 (1989) 270.

[22] T.C. Smith, 4th Int. Conf. on Ion Implantation Equipment, Berchtesgaden, FRG (Springer, Berlin, 1982).

[23] O. Puglisi, A. Licciardello, L. Calcagno and G. Foti, Nucl. Instr. and Meth. B 19/20 (1987) 865. 